\title{
Streptomycin Sulfate-Loaded Niosomes Enables Increased Antimicrobial and Anti-Biofilm Activities
}

\section{OPEN ACCESS}

Edited by:

Joao Henrique Lopes, Instituto de Tecnologia da Aeronáutica (ITA), Brazi

Reviewed by: Chunguang Yang, Institute of Metals Research (CAS),

China

Wen Shi,

University of Nebraska Medical Center, United States

${ }^{*}$ Correspondence:

Amir Mirzaie

Amir.mirzaie@piau.ac.ir

Iman Akbarzadeh Iman.akbarzadeh@che.sharif.edu

Qun Ren

Qun.Ren@empa.ch

Specialty section: This article was submitted to Biomaterials,

a section of the journal Frontiers in Bioengineering and Biotechnology

Received: 21 July 2021 Accepted: 06 September 2021 Published: 27 October 2021

Citation:

Mansouri M, Khayam N, Jamshidifar E, Pourseif T, Kianian S, Mirzaie A, Akbarzadeh I and Ren Q (2021) Streptomycin Sulfate-Loaded Niosomes Enables Increased Antimicrobial and AntiBiofilm Activities. Front. Bioeng. Biotechnol. 9:745099. doi: 10.3389/fbioe.2021.745099

\begin{abstract}
Maryam Mansouri ${ }^{1}$, Nazanin Khayam $^{2}$, Elham Jamshidifar ${ }^{3}$, Tara Pourseif ${ }^{1}$, Sepideh Kianian ${ }^{4}$, Amir Mirzaie ${ }^{5 *}$, Iman Akbarzadeh ${ }^{6 *}$ and Qun Ren ${ }^{7 *}$

${ }^{1}$ Department of Microbiology, Faculty of Advanced Science and Technology, Tehran Medical Sciences, Islamic Azad University, Tehran, Iran, ${ }^{2}$ Department of Biology, Tehran North Branch, Islamic Azad University, Tehran, Iran, ${ }^{3}$ Department of Pharmaceutical Nanotechnology, Faculty of Pharmacy, Tehran University of Medical Sciences, Tehran, Iran, ${ }^{4}$ Master of Medicinal Chemistry, Medicinal Plants and Drugs Research Institute, Shahid Beheshti University, Tehran, Iran, ${ }^{5}$ Department of Biology, Parand Branch, Islamic Azad University, Parand, Iran, ${ }^{6}$ Department of Chemical and Petrochemical Engineering, Sharif University of Technology, Tehran, Iran, ' Laboratory for Biointerfaces, Empa, Swiss Federal Laboratories for Materials Science and Technology, St. Gallen, Switzerland
\end{abstract}

One of the antibiotics used to treat infections is streptomycin sulfate that inhibits both Gram-negative and -positive bacteria. Nanoparticles are suitable carriers for the direct delivery and release of drug agents to infected locations. Niosomes are one of the new drug delivery systems that have received much attention today due to their excellent biofilm penetration property and controlled release. In this study, niosomes containing streptomycin sulfate were prepared by using the thin layer hydration method and optimized based on the size, polydispersity index (PDI), and encapsulation efficiency (EE\%) characteristics. It was found that the Span 60-to-Tween 60 ratio of 1.5 and the surfactant-to-cholesterol ratio of 1.02 led to an optimum formulation with a minimum of size, low PDI, and maximum of EE of $97.8 \mathrm{~nm}, 0.27$, and $86.7 \%$, respectively. The drug release investigation showed that $50.0 \pm 1.2 \%$ of streptomycin sulfate was released from the niosome in $24 \mathrm{~h}$ and reached $66.4 \pm 1.3 \%$ by the end of $72 \mathrm{~h}$. Two-month stability studies at $25^{\circ}$ and $4^{\circ} \mathrm{C}$ showed more acceptable stability of samples kept at $4^{\circ} \mathrm{C}$. Consequently, antimicrobial and anti-biofilm activities of streptomycin sulfate-loaded niosomes against Staphylococcus aureus, Escherichia coli, and Pseudomonas aeruginosa were found significantly higher than those of free drug, and the minimum inhibitory concentration values decreased 4- to 8-fold. Furthermore, niosomeencapsulated streptomycin up to $1,500 \mu \mathrm{g} / \mathrm{ml}$ exhibited negligible cytotoxicity against the human foreskin fibroblasts cell line, whereas the free drug exhibited slight cytotoxicity at this concentration. Desired physical characteristics and low toxicity of niosomal nanocarriers containing streptomycin sulfate made them a demanded candidate for the treatment of current bacterial infections and biofilms.

Keywords: niosome, streptomycin sulfate, antimicrobial, anti-biofilm, cytotoxicity 


\section{INTRODUCTION}

The use of drug delivery systems is essential to improve the timing, location, and speed of drug release, as well as to prevent drug fluctuations in the circulatory system that lead to lower efficacy and greater side effects (Vikas et al., 2011; Shirzad et al., 2019; Shad et al., 2020). Progress in nanotechnology led to the development of nano-carriers that are able to carry drugs to the target site (Ladavière and Gref, 2015; Heidari et al., 2020). Nanocarriers have benefits such as increased drug solubility, increased drug half-life, controlled release, targeted delivery, reduced side effects, the ability to transfer multiple drugs simultaneously, protecting the drug from degradation, and protecting the patient from immune responses to the drug (Zhang et al., 2008; Banyal et al., 2013; Ghafelehbashi et al., 2019; Akbarzadeh et al., 2021a).

One of the types of nano-carriers is niosomes, which can be an ideal choice because they are biocompatible, inert, and capable of carrying large dosage of one or more drugs (Lajevardi et al., 2018; Fang et al., 2019). They are composed of non-ionic surfactants of the class alkyl or polyglycerol diallyl ether and cholesterol, hydrated in aqueous media (Kumar and Rajeshwarrao, 2011), and can be used as carriers for low-molecular weight drugs, proteins, and genes. Due to the numerous benefits of niosomes as a drug carrier, much research has been carried out and proven to be effective for drug delivery to skin, ocular, oral, and pulmonary sites (Kumar and Rajeshwarrao, 2011; Akbarzadeh et al., 2020a; Akbarzadeh et al., 2020c). Some diseases, such as bacterial infections, require a high dosage of medication with controlled release. Also depending on the condition of these patients, it is important to have the least side effects and decrease drug resistance (Zhang et al., 2008; Banyal et al., 2013; Reta et al., 2019). Therefore, niosomal drug carriers can be a suitable candidate for infectious diseases (Akbari et al., 2013; Akbarzadeh et al., 2020c).

Infectious diseases are a major health problem and are one of the leading causes of death in developing countries. In infection, the body is invaded by pathogenic microorganisms which establish, grow, and proliferate in the host body, leading to localized cell damage, toxin secretion, or antigenic antibody responses (Brunner, 1970; Glanze et al., 1990; Bloom et al., 2000; Reta et al., 2019). Most of the commonly used antibiotics are becoming inefficient against pathogenic bacteria because of biofilm formation. Biofilms are complex structures of aggregate bacteria which are capable to survive in stressful environment conditions and cause antibiotic resistance. In recent years, different types of nanoparticles have been developed for biofilm treatment (Samiei et al., 2016; Shrestha and Kishen, 2016; Fulaz et al., 2019; Mirzaie et al., 2020).

Streptomycin is a broad-spectrum antibiotic because it kills both Gram-negative and Gram-positive bacteria and was one of the first aminoglycoside drugs discovered. It cannot be taken orally and is often prescribed as regular intramuscular injections. Aminoglycosides block the protein synthesis in the bacterium by binding to the S12 protein of the $30 \mathrm{~S}$ ribosomal unit (Judy et al., 2018); thus, they can have a bactericidal effect (Zhu et al., 2001; Sharma et al., 2007; de Lima Procópio et al., 2012).
The purpose of this investigation is developing the streptomycin sulfate-containing niosomes according to the design of experiment, followed by physicochemical characterization. The drug loading and release profiles of the streptomycin sulfate-containing niosomes were investigated. The stability of the prepared niosomes was evaluated at temperatures of $4^{\circ} \mathrm{C}$ and $25^{\circ} \mathrm{C}$ for 3 months in terms of nanoparticle size, particle size distribution (PDI), and drug encapsulation efficiency (EE\%). Finally, the antimicrobial and anti-biofilm effects of niosomes containing streptomycin sulfate on microbial strains of Staphylococcus aureus, Escherichia coli, and Pseudomonas aeruginosa were compared with the free drug.

\section{MATERIALS AND METHODS}

\section{Chemicals}

Streptomycin sulfate and phosphate buffer solution (PBS) were purchased from Bio Basic, Canada. Cholesterol, polyoxyethylene sorbitan monostearate (Tween 60), sorbitan monostearate (Span 60 ), dimethyl sulfoxide (DMSO), and chloroform were bought from Merck, Germany. 3-(4,5-dimethylthiazol-2-yl)-2,5diphenyltetrazolium bromide, penicillin/streptomycin 100X, Trypsin-EDTA, Trypan blue, RPMI 1640 medium, Dulbecco's modified Eagle medium (DMEM), fetal bovine serum (FBS) were obtained from Gibco, United States. A dialysis membrane (MWCO 12,000 Da) and MTT (dimethylthiazol-2-yl-)-2,5 were received from Sigma-Aldrich (United States). Mueller Hinton broth, Mueller Hinton agar, barium chloride, and $\mathrm{H}_{2} \mathrm{SO}_{4}$ were received from Merck, Germany. Staphylococcus aureus ATCC 6538, Escherichia coli ATCC 25922, and Pseudomonas aeruginosa ATCC 15442 were obtained from the Pasteur Institute of Iran.

\section{Preparation of Niosome}

One of the most known methods for preparing niosomes is the thin-layer hydration method (Hülsermann et al., 2009; Akbarzadeh et al., 2021b). Cholesterol and surfactants (Span 60 and Tween 60) with a 1:1 M ratio were dissolved in $10 \mathrm{ml}$ chloroform, which was evaporated using the rotary evaporator for $1 \mathrm{~h}$ at $60^{\circ} \mathrm{C}$ and $120 \mathrm{rpm}$. Afterward, the dried thin films were hydrated using streptomycin sulfate solution in PBS $(10 \mathrm{ml}$, $1.5 \mathrm{mg} / \mathrm{ml}$ ) at $30^{\circ} \mathrm{C}$ for $1 \mathrm{~h}$ with stirring at $120 \mathrm{rpm}$. Subsequently, the samples were sonicated (Hielscher UP50H ultrasonic processor, Germany) for $5 \mathrm{~min}$ and stored at $4^{\circ} \mathrm{C}$ in a refrigerator. Different formulations of niosomes were prepared, as shown in Table $\mathbf{1 .}$

\section{Optimization of Synthesized Niosome by Design of Experiments}

The purpose of applying the design of experiments is to identify the factors influencing the experiment process and determine the optimal values. The design method used in this study is D-optimal design using Design-Expert 7.0.10 software (Stat-Ease Inc., United States). This technique can identify the variables that have the most impact on output and evaluate the most optimal 
TABLE 1 | Composition of different formulations of niosomes.

\begin{tabular}{|c|c|c|c|c|c|c|}
\hline \multirow[t]{2}{*}{ Drug } & Formulation & $\begin{array}{c}\text { A: Span } \\
60: \text { Tween } 60\end{array}$ & $\begin{array}{c}\text { B: } \\
\text { Surfactant:cholesterol }\end{array}$ & $\mathbf{Z}$ average & PDI & EE \\
\hline & Unit & Molar ratio & Molar ratio & $\mathrm{nm}$ & - & $\%$ \\
\hline \multirow[t]{11}{*}{ Streptomycin sulfate (S) } & $S_{1}$ & 0.5 & 1 & 85.9 & 0.40 & 77.7 \\
\hline & $\mathrm{S}_{2}$ & 0.5 & 2 & 146.2 & 0.24 & 77.3 \\
\hline & $\mathrm{S}_{3}$ & 0.5 & 3 & 203.2 & 0.11 & 64.4 \\
\hline & $\mathrm{S}_{4}$ & 1.0 & 1 & 65.5 & 0.30 & 82.4 \\
\hline & $\mathrm{S}_{5}$ & 1.0 & 2 & 98.2 & 0.25 & 79.4 \\
\hline & $\mathrm{S}_{6}$ & 1.0 & 2 & 129.1 & 0.25 & 80.1 \\
\hline & $\mathrm{S}_{7}$ & 1.0 & 2 & 92.5 & 0.25 & 79.8 \\
\hline & $\mathrm{S}_{8}$ & 1.0 & 3 & 170.4 & 0.31 & 68.2 \\
\hline & $S_{9}$ & 1.5 & 1 & 125.0 & 0.27 & 85.7 \\
\hline & $S_{10}$ & 1.5 & 2 & 159.3 & 0.25 & 82.1 \\
\hline & $S_{11}$ & 1.5 & 3 & 362.0 & 0.29 & 71.2 \\
\hline
\end{tabular}

conditions in terms of effective factors (Gunst, 1996; BernkopSchnürch et al., 2006). For this purpose, two factors were considered: the surfactant-to-cholesterol ratio and the Span 60to-Tween $60 \mathrm{M}$ ratio as test variables, and the nanoparticle size, polydispersity index (PDI), and encapsulation efficiency (\% EE) as test responses. These variables were selected on the basis of information obtained from previous studies (Moghtaderi et al., 2021) and initial screening tests.

The morphology of optimized niosomes was characterized by the field emission scanning electron microscope (SEM). For imaging, the nanoparticle suspension was diluted 1:100 in deionized water; a drop of sample was spread on a conductor film such as aluminum and dried at room temperature.

\section{Physicochemical Characterization Particle Size}

A dynamic light dispersion analysis is a fast, non-destructive physical method used to determine the size of particles in solution and depends on the interaction of light with the particle. Therefore, Zetasizer (Malvern Instrument Ltd. Malvern, the United Kingdom), equipped with a green laser with a wavelength of $633 \mathrm{~nm}$, was used to evaluate the particle size at $25^{\circ} \mathrm{C}$. The particle size is the mean particle diameter which is represented as Z-average in nanometers. Accordingly, the more the $\mathrm{Z}$-average, the larger will be the particle size.

\section{Polydispersity Index}

The degree of particle scattering indicates the degree of dissimilarity of the particle size distribution, calculated by the Malvern nanosizer (Malvern Instrument Ltd. Malvern, United Kingdom) based on the following formula:

$$
P D I=M w / M n .
$$

\section{Encapsulation Efficiency}

To separate the free drug from the niosome-encapsulated drug, the niosomes were centrifuged at $4^{\circ} \mathrm{C}$ at $14,000 \mathrm{~g}$ for $30 \mathrm{~min}$. Through this process, the niosomes are precipitated and free drug remains in supernatant. The amount of streptomycin sulfate in the supernatant can be quantified by measuring the absorbance at $560 \mathrm{~nm}$ wavelength (Blainski et al., 2013), using a calibration curve. Finally, by applying the following formula, the percentage of encapsulation efficiency was calculated:

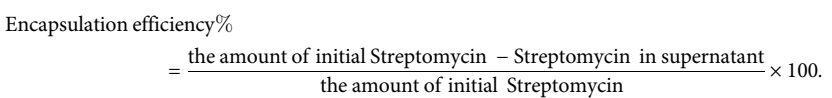

\section{In Vitro Drug Release Kinetics}

In order to evaluate the amount of drug released from niosomal carriers over a specified period of time, the dialysis membrane (molecular weight cutoff $12 \mathrm{KDa}$ ) was used to separate noisomes from the free drug. The dialysis bags containing niosomes $(2 \mathrm{ml}$ of the samples prepared earlier) or streptomycin $(1.3 \mathrm{mg} / \mathrm{ml})$ were placed in $50 \mathrm{ml}$ of PBS solution ( $\mathrm{pH} 7.4)$, which was under constant magnetic stirring at $37 \pm 1^{\circ} \mathrm{C}$. At desired time points $(1$, $2,4,8,24,48$, and $72 \mathrm{~h}$ ), $1 \mathrm{ml}$ solution was sampled and replaced with $1 \mathrm{ml}$ of fresh PBS solution. The collected sample solution was measured by colorimetric assay for the absorbance at $560 \mathrm{~nm}$ via UV-vis spectrophotometry (Jasco V-530, Japan Servo Co. Ltd., Japan) (Aman et al., 1995). To study the release kinetics and the mechanism of drug release from the niosomal formulation, the data of the drug release were mathematically analyzed based on the proportional models in kinetic models' equations, including zero-order kinetics, the Higuchi model, first-order kinetics, and the Korsmeyer-Peppas equation, by using linear form diagrams.

\section{Storage Stability Studies}

To investigate storage stability of the synthesized niosomes, $1 \mathrm{ml}$ of the streptomycin sulfate-loaded niosome solution with $1 \mathrm{mg} /$ $\mathrm{ml}$ drug concentration was poured into glass vials and stored at 4 and $25^{\circ} \mathrm{C}$, respectively, for 1 month. Samples were evaluated for the particle size and $\mathrm{EE} \%$ at different time intervals.

\section{Antimicrobial Activity}

The minimum inhibitory concentration (MIC) and minimum bactericidal concentration (MBC) were performed for empty niosome, drug-loaded niosome, and free drug using a classical microdilution method. The samples were diluted with Mueller 
Hinton broth (MHB), of which $100 \mu \mathrm{l}$ was added to a well of 96well microplate. Furthermore, the inoculums were prepared at 0.5 McFarland's standard and $50 \mu$ of selected bacterial culture including S. aureus (ATCC 6538), E. coli (ATCC 25922), and $P$. aeruginosa (ATCC 15442) was added to each well. Finally, the samples were incubated overnight at $37^{\circ} \mathrm{C}$, and the absorbance of each well was read using a microplate reader at $600 \mathrm{~nm}$ (Fard et al., 2018; Ghomi et al., 2020). The lowest concentration at which no growth was observed was considered as MIC.

To assess the MBC values, $10 \mu \mathrm{l}$ from each well was spread on Mueller Hinton agar and incubated overnight at $37^{\circ} \mathrm{C}$. Afterward, colonies were counted, and MBCs were defined as the lowest concentration of samples required to kill bacteria.

\section{Time-Kill Assay}

Antibacterial activity of free and streptomycin sulfate-loaded niosomes was determined against $S$. aureus, E. coli, and $P$. aeruginosa within $72 \mathrm{~h}$ using a microplate technique (Sadeghi et al., 2019). In brief, $100 \mu \mathrm{l}$ of the samples (free streptomycin and noisome-encapsulated streptomycin) in their sublethal concentrations (half of the MIC) were added into the 96-well microtiter plate which was preloaded with $100 \mu \mathrm{l}$ of each bacterial suspension having $10^{5} \mathrm{CFU} / \mathrm{ml}$. After incubation at $37^{\circ} \mathrm{C}$, optical density at OD $600 \mathrm{~nm}$ was measured at 2, 4, 6, 24, 48, and $72 \mathrm{~h}$ using a microplate reader (EPOCH, Japan).

\section{Anti-Biofilm Activity}

The anti-biofilm activity of free and niosome-encapsulated streptomycin sulfate against biofilms of $S$. aureus, E. coli, and $P$. aeruginosa was done using a microtiter plate-based crystal violet (CV) assay (Behdad et al., 2020). First, $180 \mu$ l of Mueller Hinton broth (MHB) culture medium and $20 \mu$ of pathogenic bacteria were added to each well to an OD 600 at 0.6 , and the mixture was incubated for $48 \mathrm{~h}$ at $37^{\circ} \mathrm{C}$ at $120 \mathrm{rpm}$ to allow biofilm formation. Then $100 \mu \mathrm{l}$ of niosomal streptomycin and free streptomycin at the MIC level, free niosome, and free MHB medium (negative control) were added. The plates were then incubated at $37^{\circ} \mathrm{C}$ for $24 \mathrm{~h}$. Afterward, the supernatants were removed and the wells washed with $300 \mu$ PBS to remove nonadherent cells from the wells. The plates were then air-dried. The biofilms were fixed with $175 \mu \mathrm{l} 2 \%$ sodium acetate and stained with $175 \mu \mathrm{l} 0.1 \%$ violet crystal for $30 \mathrm{~min}$ in the dark. The wells were then washed with PBS to remove excess dye. Finally, $200 \mu \mathrm{l}$ of ethanol was added to the wells, and their absorption was read at $570 \mathrm{~nm}$.

\section{Cytotoxicity Study}

To investigate cytotoxicity of free streptomycin sulfate, free niosome, and streptomycin sulfate-loaded niosome towards the human foreskin fibroblast (HFF) normal cell line, the colorimetric MTT [(3-(4, 5-dimethylthiazol-2-yl)-2, 5diphenyl-tetrazolium bromide] assay was used. In brief, the HFF cells were seeded into 96-well plates for $24 \mathrm{~h}$ at $37^{\circ} \mathrm{C}$. Then, various concentrations of free streptomycin sulfate, free niosome, and streptomycin sulfate-loaded niosome were added into each well. After incubation time, $100 \mu \mathrm{l}$ of MTT dye was added to the wells and incubated for $4 \mathrm{~h}$ at $37^{\circ} \mathrm{C}$. Subsequently,
$100 \mu \mathrm{l}$ of DMSO was added, and the absorbance was measured at $570 \mathrm{~nm}$ using a microplate reader (AccuReader, Metertech, Taiwan), and the cell survival rate was calculated by the formula:

Cell viability $(\%)=$ Optical density of sample/Optical density of control $\times 100$.

For control, HFF cells were incubated with the Dulbecco's modified Eagle medium (DMEM) without the test sample (Ali et al., 2010).

\section{Statistical Analysis}

A Statistical analysis was performed using Design-Expert 7.0.10 software (Stat-Ease Inc., United States) and IBM SPSS Statistics version 24. ANOVA was used to compare multiple samples, and the $p$ value $<0.05$ was considered significant.

\section{RESULTS AND DISCUSSION}

\section{Niosomal Formulations and Physicochemical Properties}

In the structure of the niosomes, the size of the vesicles and the efficiency of streptomycin sulfate encapsulation are highly dependent on the type of surfactant and the ratio of surfactant to cholesterol. For a suitable drug delivery system, it is desired to have a small size and a high encapsulation efficiency (EE) (Moghaddam et al., 2021; Moghtaderi et al., 2021). A variety of niosomal formulations synthesized with different ratios of surfactant to cholesterol and Span 60 to Tween 60 were prepared and compared (Table 1). It was found that with the same Span 60 -to-Tween 60 ratio, an increasing surfactant-to-cholesterol ratio led to a larger nano-vesicle size and lower EE. The minimum size was at low levels of the Span 60-to-Tween 60 and surfactant-to-cholesterol ratios as shown in the threedimensional graph (Figure 1A). The maximum EE of nanovesicles was found at the high Span 60-to-Tween 60 ratio and low levels of the surfactant-to-cholesterol ratio (Figure 1B). The lowest PDI was found at low Span 60-to-Tween 60 and high surfactant-to-cholesterol ratios (Figure 1C).

As mentioned, Span and Tween are non-ionic surfactants that have many advantages such as improved stability, broad compatibility, and flexibility of formulation. Due to the weak rigidity of Tween 60 and the high lipophilicity of Span 60, proper confinement of cholesterol and surfactant (Span 60:Tween 60) in a 1:1 M ratio can lead to the density of niosome films (Junyaprasert et al., 2012; Ghafelehbashi et al., 2019). By the combination of Span and Tween in different ratios, systems with a wide range of hydrophobic-lipophilic balance (HLB) are produced. In addition, studies have shown that high transition temperatures of Span and Tween 60 provide high levels of drug encapsulation (Bharti et al., 2012; Taymouri and Varshosaz, 2016). Increasing the amount of cholesterol increases the lipid profile and stability of the two layers and results in reduced permeability, so that the drug can be trapped more effectively in the vesicles. However, excessive amounts of cholesterol make the drug and cholesterol compete for the space between the two layers, and consequently, the drug cannot enter the structure (Balakrishnan et al., 2009). It was reported that a 1:1 M ratio of 

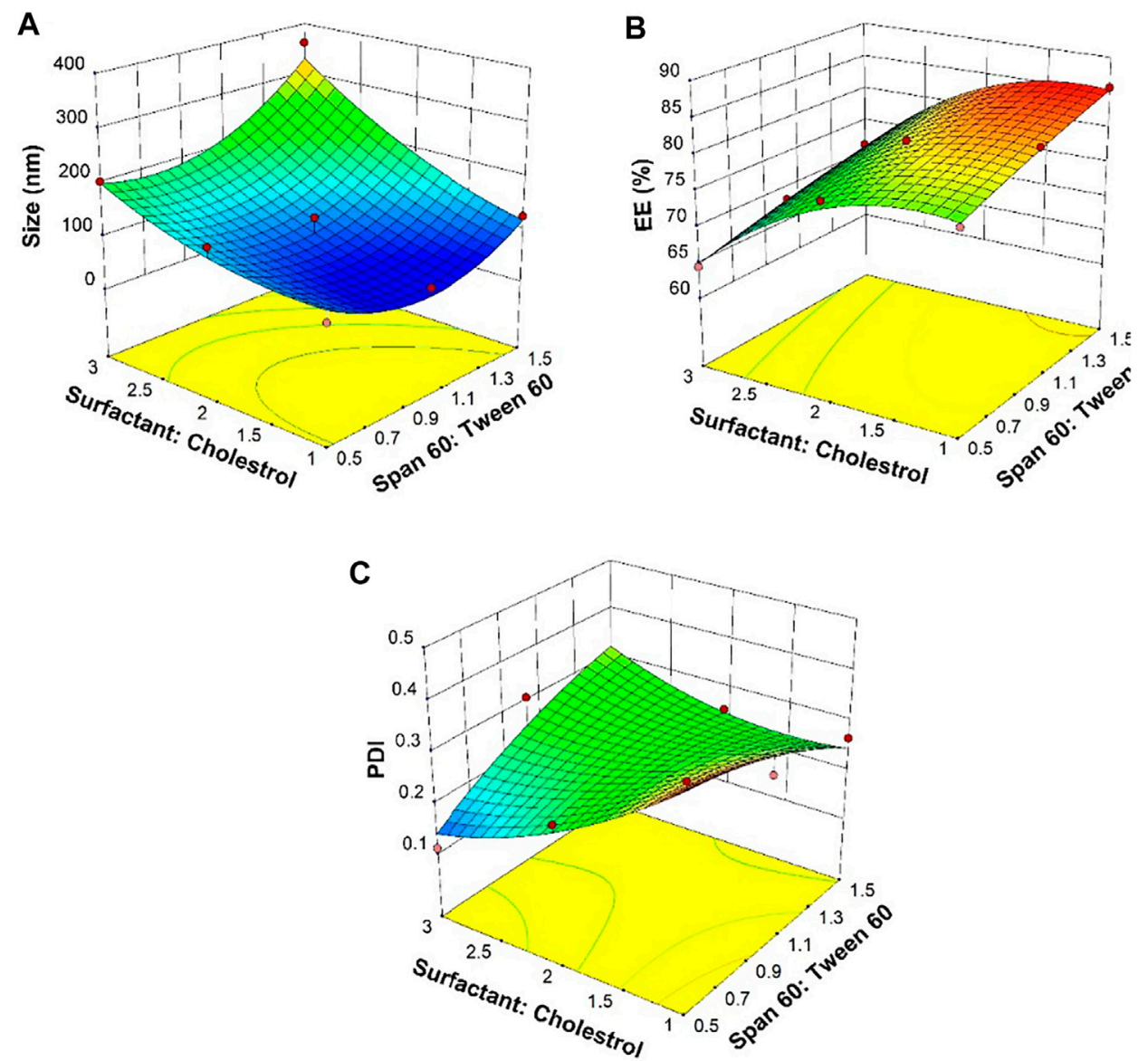

FIGURE 1 | Response surfaces for (A) average diameter, (B) entrapment efficiency (EE), and (C) polydispersity index (PDI) as an outcome of Span 60 -to-Tween 60 and surfactant-to-cholesterol molar ratios.

TABLE 2 | Desirability criteria and predicted values for independent variables.

\begin{tabular}{cccc} 
Number & Span 60:Tween $\mathbf{6 0}$ & Surfactant:cholesterol & Desirability \\
\hline 1 & 1.50 & 1.02 & 0.80
\end{tabular}

cholesterol to surfactant results in a high EE formulation (Rochdy Haj-Ahmad et al., 2015). The PDI value is an estimate of the particle distribution and their heterogeneity, which is measured between 0 and 1 (Moghassemi et al., 2015). The uniform particles have better distribution and less tendency to accumulate (Waddad et al., 2013).

Based on the data shown in Table 2, multi-criteria optimization was performed by using the desirability function to obtain the optimal formulation (Derringer and Suich, 1980; Esfahani et al., 2019; Shabani et al., 2020). According to the desired parameters, the predicted optimal formulation was calculated and compared with the experimentally obtained one (Table 3). It was found that both predicted and empirically obtained formulation showed similar values, with the latter having a size of $97.8 \mathrm{~nm}$, a polydisperse index of 0.27 , and an encapsulation efficiency of $86.7 \%$.
TABLE 3 | Comparison of the empirical and predicted values for the optimized niosomal formulation.

\begin{tabular}{lccc}
\hline Source & Z-average & PDI & EE \\
\hline Predicted & 118.8 & 0.25 & 85.5 \\
Empirical & $97.8 \pm 5.0$ & $0.27 \pm 0.03$ & $86.7 \pm 1.1$
\end{tabular}

\section{Morphological Characterization}

A field emission scanning electron microscope (FE-SEM) was used to investigate the morphology of the synthesized niosomes. It was observed that the streptomycin sulfate-containing niosomes were perfectly spherical in morphology, with a smooth surface (Figure 2). The average particle size for the synthesized niosomes is approximately $20-40 \mathrm{~nm}$, which is less than the size obtained by the light scattering method. This difference could be because the FE-SEM shows the nanoparticle size in the dried form (actual nanoparticle size), while DLS measures the hydrodynamic diameter, which may include any molecule (such as like ions or water molecules) attached to the nanoparticle surface.

Multilayer niosomes were observed using an FE-SEM, which has been reported previously. Niosomes prepared by thin-layer 


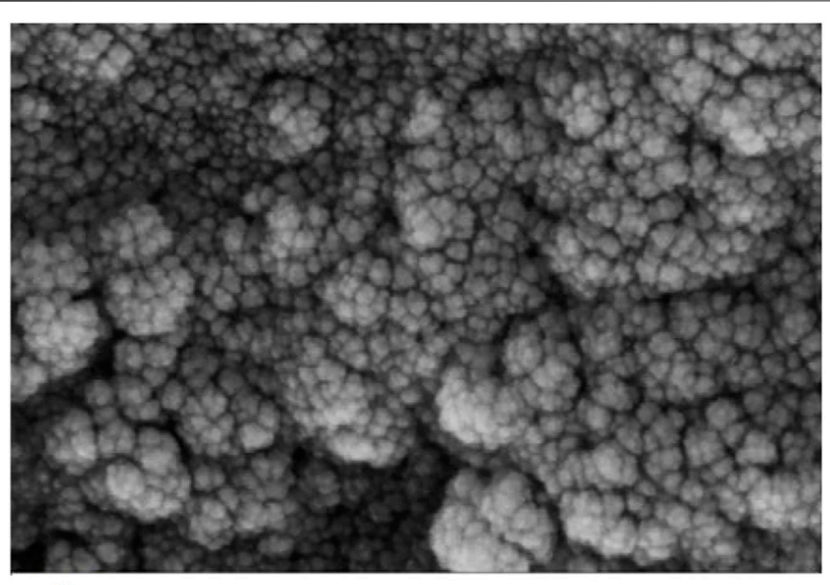

$100 \mathrm{~mm}$

FIGURE 2 | Morphological characterization of optimized niosomes by SEM.

hydration is usually multilayer vesicles below $100 \mathrm{~nm}$ with suitable particle distribution that can confine a large amount of drug and slower drug release (Hope et al., 1986; Akbari et al., 2015; El-Sayed et al., 2017).

\section{Stability Study}

During storage, the niosomes can swell/break down or are affected by steric/repulsion forces. Here, we investigated the stability of the synthesized niosomes at 4 and $25^{\circ} \mathrm{C}$ for 60 days. It was found that samples stored at $4^{\circ} \mathrm{C}$ had better stability in terms of size, PDI, and EE than those stored at $25^{\circ} \mathrm{C}$ during the 60 -day storage (Figure 3). There was a significant difference in the size of the niosomes kept at two temperatures, and the size increase for the samples stored at $4^{\circ} \mathrm{C}$ was slower than the corresponding sample at $25^{\circ} \mathrm{C}$, which could be due to less mobility of the niosomes at $4^{\circ} \mathrm{C}$ (Lawrence et al., 1996; Balasubramaniam et al., 2002; Akbarzadeh et al., 2020b). Studies have also shown that the size of formulations can

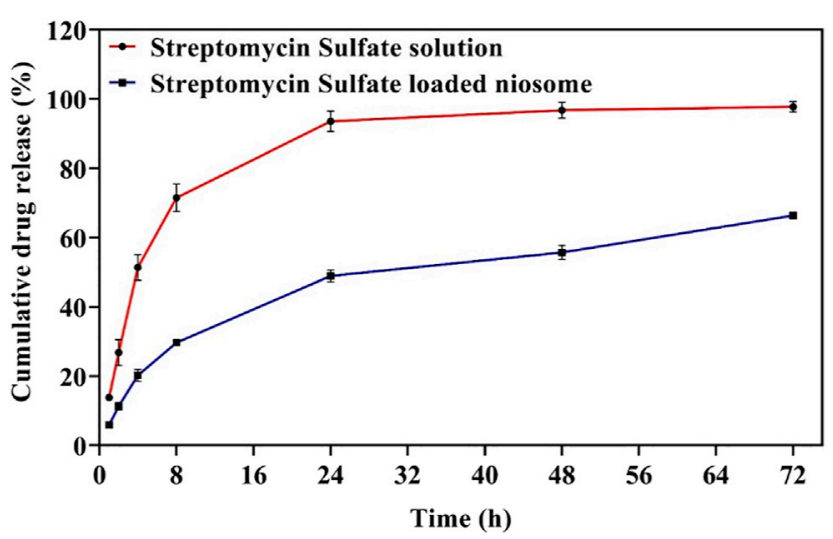

FIGURE 4 | The release profile of free and optimized niosomal formulation of streptomycin sulfate. Each point represents the mean $\pm \mathrm{SD}$ $(n=3)$.

affect the stability of the system because, according to the theory of thermodynamics, smaller niosomes contain excess energy, which makes them unstable. The high leakage of the drug at $25^{\circ} \mathrm{C}$ can also be caused by the higher fluidity of the lipid vesicles at high temperature (Pardakhty et al., 2011; Akbarzadeh et al., 2020b; Akbarzadeh et al., 2020c).

\section{In Vitro Drug Release}

The drug release rate is an essential factor for upgrading drug delivery systems. The release of encapsulated drugs within the niosomes can be optimized for controlled drug release over the long term (Tarekegn et al., 2010). Here, we compared the release profile of the streptomycin sulfate-soluble form and the encapsulated niosome form in the PBS medium for $72 \mathrm{~h}$. It was found that the streptomycin sulfate release from the nano-carrier $(66.4 \pm 1.3 \%)$ was lower than the drug solution $(97.8 \pm 1.12 \%)$ during $72 \mathrm{~h}$ of release (Figure 4). Thus, encapsulation of streptomycin sulfate in the niosome reduced the release burst and allowed more sustainable and
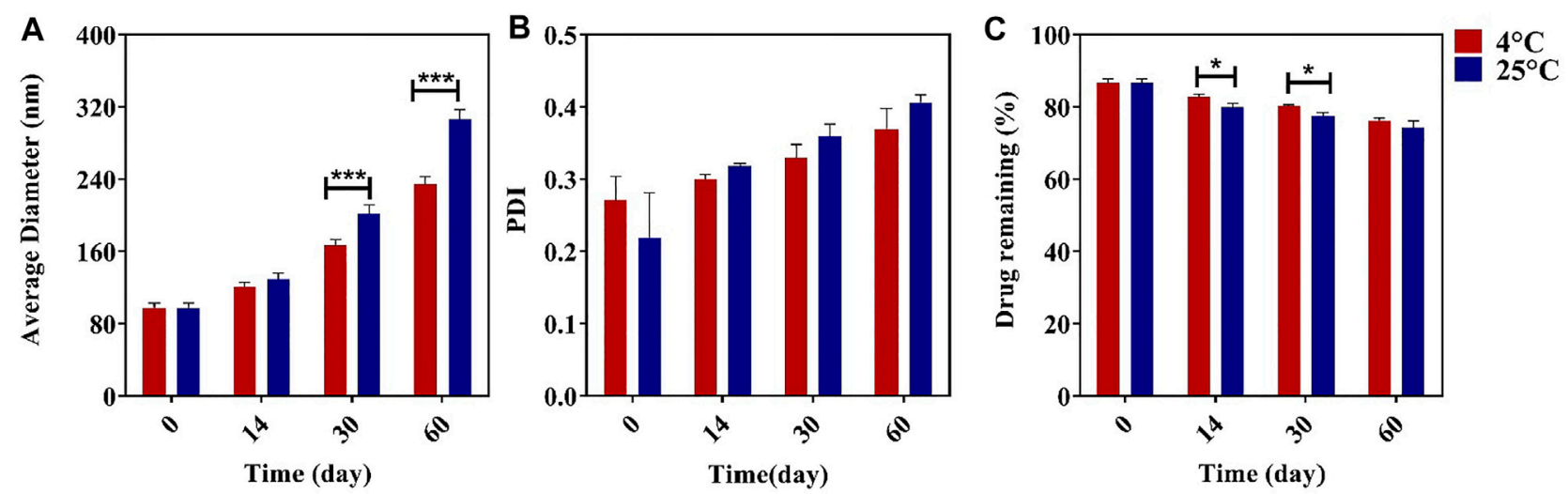

FIGURE 3 | Effect of different temperatures of storage on the average diameter (A), polydispersity index (PDI) (B), and streptomycin sulfate encapsulation efficiency (EE\%) (C) $n=3,{ }^{*} p$ value $<0.05,{ }^{\star \star} p$ value $<0.01$, and ${ }^{\star \star \star} p$ value $<0.001$ 
TABLE 4 | Release kinetic models and the parameters obtained for niosomal formulations.

Release model

Equation
Streptomycin sulfate solution

$\boldsymbol{R}^{2}$

$R^{2}=0.62$

$R^{2}=0.85$

$n=0.43$

$R^{2}=0.88$

$R^{2}=0.79$
Streptomycin

sulfate-loaded niosome

$\log C=\log C 0+K_{t} / 2.303$

Zero-order

First-order

$\mathrm{Q}=\mathrm{K}_{\mathrm{H}} \sqrt{ } t$

$\mathrm{C}_{\mathrm{t}}=\mathrm{C}_{0}+\mathrm{K}_{0} \mathrm{t}$

Higuch

${ }^{a}$ Diffusion or release exponent.
A $\square$ Free streptomycin sulfate

Streptomycin sulfate loaded niosome

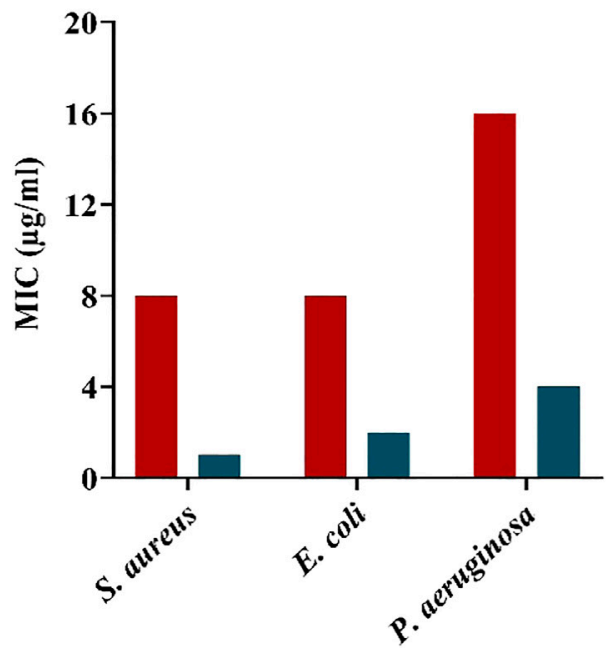

B $\square$ Free streptomycin sulfate

Streptomycin sulfate loaded niosome

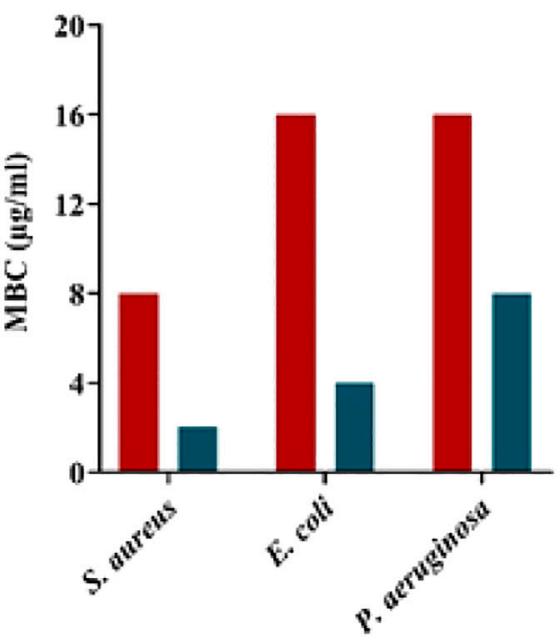

FIGURE 5 | MIC (A) and MBC (B) of free and niosome-encapsulated streptomycin sulfate. $n=3$.
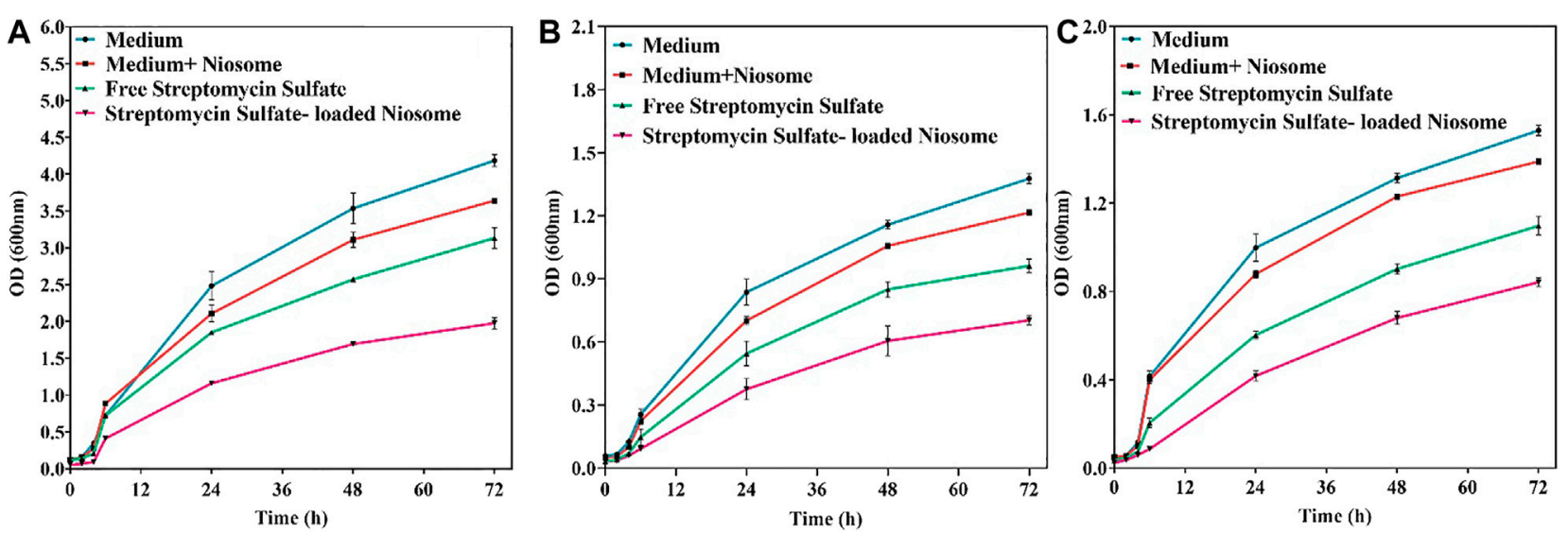

FIGURE 6 | Antibacterial activity of free streptomycin and encapsulated streptomycin against pathogenic bacteria: S. aureus (A), E. coli (B), and P. aeruginosa (C) measured by optical density as a function of time $(72 \mathrm{~h})$. Each point corresponds to a mean \pm SD with three replicates per condition. 


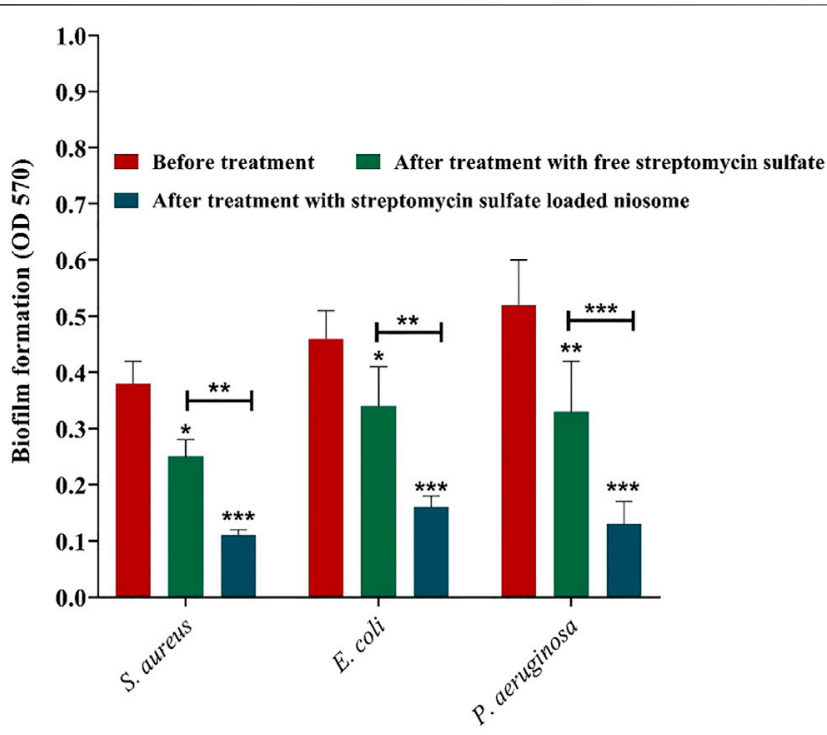

FIGURE 7 | Anti-biofilm activity of free and niosome-encapsulated streptomycin sulfate against selected pathogenic bacterial biofilms at their minimal inhibition concentrations shown in Figure 5. Biofilms were formed in a 96-well microplate and, consequently, treated for $24 \mathrm{~h}$ at $37^{\circ} \mathrm{C}$. The remaining biofilm was quantified by $\mathrm{CV}$ staining and compared with the untreated one. Data represent the mean $\pm \operatorname{SD}(n=3)$. Error bars represent standard deviations. The levels of significant difference are denoted by ${ }^{*} p$ value $<0.05,{ }^{* \star} p$ value $<0.01$, and ${ }^{* \star *} p$ value $<0.001$

prolonged release. It has been previously reported that the release profile of niosomes can have two steps: the first is faster and the second is slower (Paolino et al., 2008; Akbarzadeh et al., 2020c). The rapid release of the drug in the first stage is due to the excretion of the drug from the outer surface of the niosome, and the slower release in the second stage is due to the penetration of the drug through the niosome (Manosroi and Bauer, 1989). Other factors contributing to the release can be components of the niosomes. As the amount of cholesterol in the niosomes increases, the amount of drug release from the vesicles decreases because, at higher cholesterol levels, the movement of the bilayer cannot eliminate osmotic changes and lead to the released drugs absorbed to the niosomal surface (Liang et al., 2004; Ruckmani and Sankar, 2010; Hedayati Ch et al., 2020; Sadeghi et al., 2020). In addition, as the surfactant chain length increases, drug release can continue for a longer period because the transfer temperature can affect the surfactants and make them completely fluid, thereby providing greater penetration of the drug at $37^{\circ} \mathrm{C}$ (Ruckmani et al., 2000; El-Ridy et al., 2018).

Different models were evaluated to fit the release kinetics of streptomycin sulfate from the optimal niosomal formulation (Table 4). Based on the model parameters and the coefficient of determination (R2) for each model, the release for niosomal formulation was found to follow the Korsmeyer-Peppas model, where the $\mathrm{N}$ obtained values $(n<0.45)$ indicate that the Fickian diffusion mechanism determines the release of streptomycin sulfate molecules from the niosomal formulation (Korsmeyer et al., 1983).

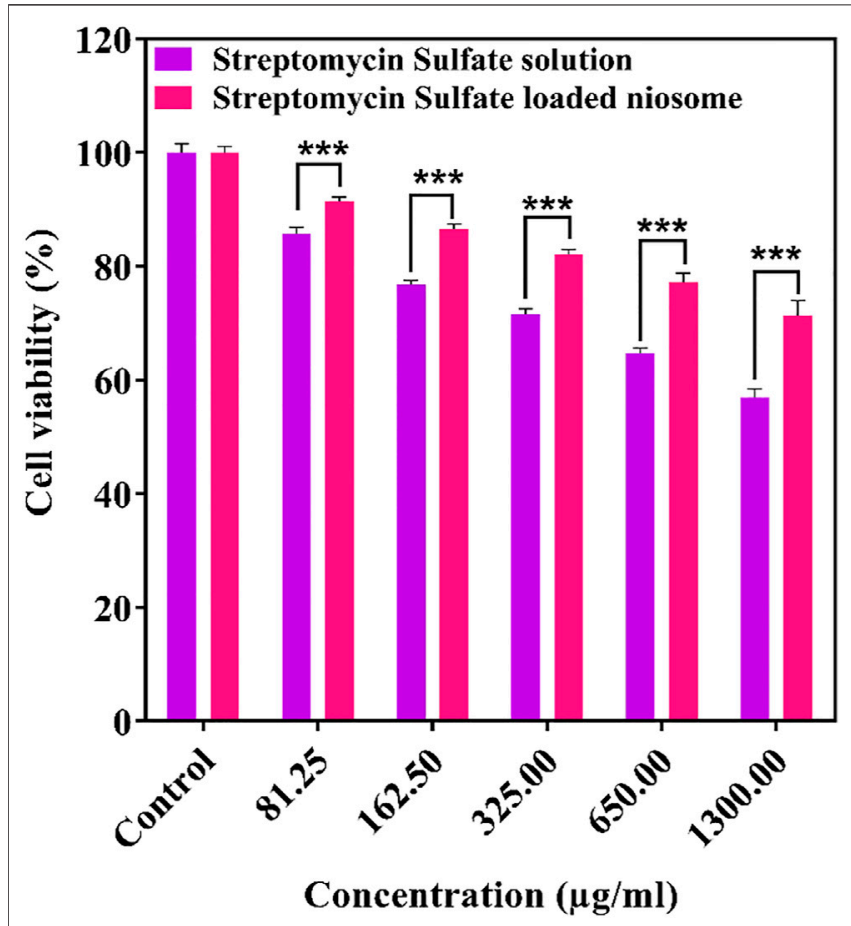

FIGURE 8 | Cytotoxicity of free and niosomal streptomycin sulfate in different concentration against HFF after 24 h. ${ }^{\star \star \star} p<0.001,{ }^{\star \star} p<0.01$, ${ }^{*} p<0.05$

\section{Antimicrobial Activity}

We further investigated the antimicrobial activity of the synthesized niosomes by measuring minimum inhibitory concentration (MIC) and minimum bactericidal concentration (MBC). Free niosome, free streptomycin sulfate, and streptomycin sulfate-loaded niosomes against $S$. aureus, $E$. coli, and $P$. aeruginosa were tested. The streptomycin sulfate-loaded niosomes showed a higher antibacterial effect against all studied pathogenic bacteria than free streptomycin sulfate, with the MIC values decreased between 4- and 8-fold (Figure 5). Furthermore, lower MBC was found for the streptomycin sulfate-containing niosomes than for the free streptomycin sulfate. These results suggest that lower concentrations of niosomal streptomycin sulfate are needed to inhibit bacterial growth than free streptomycin sulfate. This could be caused by the possibility that niosomes can protect drug against the effects of bacterial enzymes and facilitate niosome fusion with the bacterial membrane, as reported previously (Mugabe et al., 2005; Moammeri et al., 2021; Moghtaderi et al., 2021).

To investigate the killing profile of the synthesized niosomes, we next performed the time-kill assay against $S$. aureus, E. coli, and $P$. aeruginosa with sublethal concentrations (half of the MIC shown in Figure 5). During the 72-h test, the loaded niosomes displayed highest antibacterial activity compared to the unloaded niosomes and free streptomycin (Figure 6). The results further demonstrate that the direct interaction of the niosomal carrier with bacteria (likely cell membrane) could be a reason for the 
greater antibacterial property in the niosomes, as reported previously (Zille et al., 2015; Raza et al., 2016; Ghafelehbashi et al., 2019; Moghtaderi et al., 2021).

\section{Anti-Biofilm Activity}

Since biofilm is a prevalent factor causing antimicrobial resistance and accounts for $65-80 \%$ of all infections (Macià et al., 2018), the fabricated niosomes here were investigated for their efficacy against biofilms of $S$. aureus, E. coli, and $P$. aeruginosa with MIC shown in Figure 5. It was revealed that streptomycin-loaded niosomes reduced significantly the preformed biofilm in comparison to the free streptomycin (Figure 7). Previously, it has been reported that niosomal vesicles, due to their cationicity, interact electrostatically with the negatively charged biofilms; the drug can be released into the biofilm structure. Thus, niosomes are excellent carriers for delivery of antimicrobial drugs for eradication of biofilms. Previously, Kashef et al. studied the anti-biofilm effects of ciprofloxacin-containing niosomes against $S$. aureus biofilm and showed that niosome encapsulation reduced the minimum biofilm eradication concentration of ciprofloxacin by 2 - to 4 -fold compared to free ciprofloxacin (Kashef et al., 2020). In this work, even with 4- to 8-fold lower MIC of the free streptomycin (Figure 5), encapsulation allowed more efficient removal of the biofilm (Figure 7). This result once more demonstrates the power of niosomes.

\section{Cytotoxicity}

The free and encapsulated streptomycin was evaluated for their cytotoxicity toward the HFF using the MTT assay. The cells exposed to medium only were used as control, and their viability was set to $100 \%$. The cytotoxic cutoff was set as $70 \%$ of the viable cells in the control. It can be noticed that the niosome-encapsulated streptomycin had low toxicity (cell viability above $70 \%$ ) to the HFF cells within the tested concentrations up to $1,300 \mu \mathrm{g} / \mathrm{ml}$ after $24 \mathrm{~h}$, whereas free streptomycin exhibited toxicity with a concentration at $1,300 \mu \mathrm{g} / \mathrm{ml}$ (Figure 8). The lower toxicity of the niosomeencapsulated streptomycin than the free drug may be due to the use of surfactants (Span and Tween) in the niosomes, which are highly biocompatible (Marianecci et al., 2010; Shaker et al., 2015). The lower toxicity can also be caused by the lower release of streptomycin from niosome than the free form (Figure 4): after 24-h interaction, only about half of streptomycin was released from niosomes compared with that from the free form. Consequently, slightly higher cytotoxicity

\section{REFERENCES}

Akbari, V., Abedi, D., Pardakhty, A., and Sadeghi-Aliabadi, H. (2013). Ciprofloxacin Nano-Niosomes for Targeting Intracellular Infections: an In Vitro Evaluation. J. Nanopart Res. 15 (4), 1556. doi:10.1007/s11051-013-1556-y

Akbari, V., Abedi, D., Pardakhty, A., and Sadeghi-Aliabadi, H. (2015). Release Studies on Ciprofloxacin Loaded Non-ionic Surfactant Vesicles. Avicenna J. Med. Biotechnol. 7 (2), 69-75.

Akbarzadeh, I., Fatemizadeh, M., Heidari, F., and Niri, N. M. (2020a). Niosomal Formulation for Co-administration of Hydrophobic Anticancer Drugs into was observed for the free form than the niosome form (Figure 8), with about $70 \%$ cell viability for the former and $80 \%$ for the latter at the highest tested concentration. The low toxicity of niosomal carriers can be an ideal proposition in clinical applications.

\section{CONCLUSION}

In this study, the optimal niosomal formulation was designed and synthesized for streptomycin sulfate, with the highest encapsulation efficiency but a minimum size and low PDI. The optimized niosomal formulation exhibited controlled drug release and antibacterial effects against both Gram-positive and -negative strains. In addition to increased antibacterial activity of drug-containing nano-carriers, the niosomes showed reduced toxicity to normal cells compared to free streptomycin sulfate. The results of this study can lead to a new therapeutic process in the improvement and treatment of infection. It is envisaged that further in vivo studies shall be performed to investigate the function of this nanostructure in treatment of microbial infections.

\section{DATA AVAILABILITY STATEMENT}

The original contributions presented in the study are included in the article/Supplementary Material; further inquiries can be directed to the corresponding authors.

\section{AUTHOR CONTRIBUTIONS}

IA, AM, and $\mathrm{QR}$ developed the idea and designed the experiments. MM, IA, TP, and AM conducted the experiments. NK, EJ, and SK analyzed the data. MM wrote the manuscript, and QR performed validation and writing-review and editing of the manuscript (Supervision). All authors confirmed the final manuscript before the submission.

\section{ACKNOWLEDGMENTS}

The authors would like to acknowledge Islamic Azad University, Tehran Medical Branch, for providing the necessary laboratory facilities for this study.

MCF-7 Cancer Cells. Arch. Adv. Biosci. 11 (2), 1-9. doi:10.22037/ aab.v11i2.28906

Akbarzadeh, I., Saremi Poor, A., Yaghmaei, S., Norouzian, D., Noorbazargan, H., Saffar, S., et al. (2020b). Niosomal Delivery of Simvastatin to MDA-MB-231 Cancer Cells. Drug Dev. Ind. Pharm. 46, 1535-1549. (just-accepted). doi:10.1080/03639045.2020.1810269

Akbarzadeh, I., Yaraki, M. T., Bourbour, M., Noorbazargan, H., Lajevardi, A., Shilsar, S. M. S., et al. (2020c). Optimized Doxycycline-Loaded Niosomal Formulation for Treatment of Infection-Associated Prostate Cancer: An InVitro Investigation. J. Drug Deliv. Sci. Technol. 57, 101715. doi:10.1016/ j.jddst.2020.101715 
Akbarzadeh, I., Keramati, M., Azadi, A., Afzali, E., Shahbazi, R., chiani, M., et al. (2021a). Optimization, Physicochemical Characterization, and Antimicrobial Activity of a Novel Simvastatin Nano-Niosomal Gel against E. coli and S. aureus. Chem. Phys. Lipids 234, 105019. doi:10.1016/j.chemphyslip.2020.105019

Akbarzadeh, I., Shayan, M., Bourbour, M., Moghtaderi, M., Noorbazargan, H., Eshrati Yeganeh, F., et al. (2021b). Preparation, Optimization and In-Vitro Evaluation of Curcumin-Loaded Niosome@calcium Alginate Nanocarrier as a New Approach for Breast Cancer Treatment. Biology 10 (3), 173. doi:10.3390/ biology10030173

Ali, H., Shirode, A. B., Sylvester, P. W., and Nazzal, S. (2010). Preparation, Characterization, and Anticancer Effects of Simvastatin-Tocotrienol Lipid Nanoparticles. Int. J. Pharm. 389 (1-2), 223-231. doi:10.1016/ j.ijpharm.2010.01.018

Aman, T., Rashid, A., Kulsoom, R., and Khokhar, I. (1995). Spectrophotometric Determination of Streptomycin. Anal. Lett. 28 (5), 881-892. doi:10.1080/ 00032719508001431

Balakrishnan, P., Shanmugam, S., Lee, W. S., Lee, W. M., Kim, J. O., Oh, D. H., et al. (2009). Formulation and In Vitro Assessment of Minoxidil Niosomes for Enhanced Skin Delivery. Int. J. Pharm. 377 (1-2), 1-8. doi:10.1016/ j.ijpharm.2009.04.020

Balasubramaniam, A., Anil Kumar, V., and Sadasivan Pillai, K. (2002). Formulation and In Vivo Evaluation of Niosome-Encapsulated Daunorubicin Hydrochloride. Drug Dev. Ind. Pharm. 28 (10), 1181-1193. doi:10.1081/ddc-120015351

Banyal, S., Malik, P., Tuli, H. S., and Mukherjee, T. K. (2013). Advances in Nanotechnology for Diagnosis and Treatment of Tuberculosis. Curr. Opin. Pulm. Med. 19 (3), 289-297. doi:10.1097/MCP.0b013e32835eff08

Behdad, R., Pargol, M., Mirzaie, A., Karizi, S. Z., Noorbazargan, H., and Akbarzadeh, I. (2020). Efflux Pump Inhibitory Activity of Biologically Synthesized Silver Nanoparticles against Multidrug-Resistant Acinetobacter Baumannii Clinical Isolates. J. Basic Microbiol. 60 (6), 494-507. doi:10.1002/ jobm.201900712

Bernkop-Schnürch, A., Weithaler, A., Albrecht, K., and Greimel, A. (2006). Thiomers: Preparation and In Vitro Evaluation of a Mucoadhesive Nanoparticulate Drug Delivery System. Int. J. pharm. 317 (1), 76-81. doi:10.1016/j.ijpharm.2006.02.044

Bharti, N., Loona, S., and Khan, M. (2012). Pro-niosomes: a Recent Advancement in Nanotechnology as a Drug Carrier. Int. J. Pharm. Sci. Rev. Res. 12, 67-75. doi:10.1016/s1473-3099(12)70005-1

Blainski, A., Lopes, G., and De Mello, J. (2013). Application and Analysis of the Folin Ciocalteu Method for the Determination of the Total Phenolic Content from Limonium Brasiliense L. Molecules 18 (6), 6852-6865. doi:10.3390/ molecules 18066852

Bloom, B. R., Cohen, M. L., Donnenberg, M. S., Walsh, C., Aderem, A., Ulevitch, R. J., et al. (2000). Microbial Infection \& Immune Defence. Nature 406 (6797), 759. doi:10.1038/35021201

Brunner, L. S. (1970). Textbook of Medical-Surgical Nursing. Philadelphia, PA: Lippincott.

Derringer, G., and Suich, R. (1980). Simultaneous Optimization of Several Response Variables. J. Qual. Technol. 12 (4), 214-219. doi:10.1080/ 00224065.1980.11980968

El-Ridy, M. S., Yehia, S. A., Mohsen, A. M., El-Awdan, S. A., and Darwish, A. B. (2018). Formulation of Niosomal Gel for Enhanced Transdermal Lornoxicam Delivery: In-Vitro and In-Vivo Evaluation. Curr. Drug Deliv. 15 (1), 122-133. doi:10.2174/1567201814666170224141548

El-Sayed, M. M., Hussein, A. K., Sarhan, H. A., and Mansour, H. F. (2017). Flurbiprofen-loaded Niosomes-In-Gel System Improves the Ocular Bioavailability of Flurbiprofen in the Aqueous Humor. Drug Dev. Ind. Pharm. 43 (6), 902-910. doi:10.1080/03639045.2016.1272120

Esfahani, T. H., Azadi, A., Joodi, N., Cohan, R. A., Akbarzadeh, I., and Bakhshandeh, H. J. H. B. B. (2019). Optimized Preparation of Lysozyme Loaded Dextran-Chitosan Nanoparticles Using D-Optimal Design. Heath biotechnol. biopharma. 2, 56-78.

Fang, Z., Pan, S., Gao, P., Sheng, H., Li, L., Shi, L., et al. (2019). Stimuli-responsive Charge-Reversal Nano Drug Delivery System: the Promising Targeted Carriers for Tumor Therapy. Int. J. Pharm. 575, 118841. doi:10.1016/j.jpharm.2019.118841

Fard, N. N., Noorbazargan, H., Mirzaie, A., Hedayati Ch, M., Moghimiyan, Z., and Rahimi, A. (2018). Biogenic Synthesis of AgNPs Using Artemisia Oliveriana
Extract and Their Biological Activities for an Effective Treatment of Lung Cancer. Artif. Cell nanomed. Biotechnol. 46 (Suppl. 3), S1047-S1058. doi:10.1080/21691401.2018.1528983

Fulaz, S., Vitale, S., Quinn, L., and Casey, E. (2019). Nanoparticle-Biofilm Interactions: The Role of the EPS Matrix. Trends Microbiol. 27 (11), 915-926. doi:10.1016/j.tim.2019.07.004

Ghafelehbashi, R., Akbarzadeh, I., Tavakkoli Yaraki, M., Lajevardi, A., Fatemizadeh, M., and Heidarpoor Saremi, L. (2019). Preparation, Physicochemical Properties, In Vitro Evaluation and Release Behavior of Cephalexin-Loaded Niosomes. Int. J. pharm. 569, 118580. doi:10.1016/j.ijpharm.2019.118580

Ghomi, Z., Tafvizi, F., Naseh, V., and Akbarzadeh, I. (2020). Effect of Artemisia Ciniformis Extract on Expression of NorA Efflux Pump Gene in Ciprofloxacin Resistant Staphylococcus aureus by Real Time PCR. Iran J. Med. Microbiol. 14 (1), 55-69. doi:10.30699/ijmm.14.1.55

Glanze, W. D., Anderson, K., and Anderson, L. E. (1990). Mosby's Medical, Nursing, and Allied Health Dictionary. Mosby.

Gunst, R. F. (1996). Response Surface Methodology: Process and Product Optimization Using Designed Experiments. Taylor \& Francis.

Haj-Ahmad, R., Elkordy, A., and Chaw, C. (2015). In Vitro characterisation of $\operatorname{Span}^{\mathrm{TM}} 65$ Niosomal Formulations Containing Proteins. Cdd 12 (5), 628-639. doi:10.2174/1567201812666150511095432

Hedayati Ch, M., Abolhassani Targhi, A., Shamsi, F., Heidari, F., Salehi Moghadam, Z., Mirzaie, A., et al. (2020). Niosome-encapsulated Tobramycin Reduced Antibiotic Resistance and Enhanced Antibacterial Activity against Multidrug-resistant Clinical Strains of Pseudomonas aeruginosa. J. Biomed. Mater. Res. A 109, 966-980. doi:10.1002/jbm.a.3708

Heidari, F., Akbarzadeh, I., Nourouzian, D., Mirzaie, A., and Bakhshandeh, H. (2020). Optimization and Characterization of Tannic Acid Loaded Niosomes for Enhanced Antibacterial and Anti-biofilm Activities. Adv. Powder Technol. 31 (12), 4768-4781. doi:10.1016/j.apt.2020.11.008

Hope, M., Bally, M., Mayer, L., Janoff, A., and Cullis, P. (1986). Generation of Multilamellar and Unilamellar Phospholipid Vesicles. Chem. Phys. Lipids 40 (24), 89-107. doi:10.1016/0009-3084(86)90065-4

Hülsermann, U., Hoffmann, M. M., Massing, U., and Fricker, G. (2009). Uptake of Apolipoprotein E Fragment Coupled Liposomes by Cultured Brain Microvessel Endothelial Cells and Intact Brain Capillaries. J. Drug Target. 17 (8), 610-618. doi:10.1080/10611860903105986

Judy, E., Pagariya, D., and Kishore, N. (2018). Drug Partitioning in Micellar Media and its Implications in Rational Drug Design: Insights with Streptomycin. Langmuir 34 (11), 3467-3484. doi:10.1021/acs.langmuir.7b04346

Junyaprasert, V. B., Singhsa, P., Suksiriworapong, J., and Chantasart, D. (2012). Physicochemical Properties and Skin Permeation of Span 60/Tween 60 Niosomes of Ellagic Acid. Int. J. Pharm. 423 (2), 303-311. doi:10.1016/j.ijpharm.2011.11.032

Kashef, M. T., Saleh, N. M., Assar, N. H., Ramadan, M. A., and Resistance, D. (2020). The Antimicrobial Activity of Ciprofloxacin-Loaded Niosomes against Ciprofloxacin-Resistant and Biofilm-Forming Staphylococcus aureus. Idr 13, 1619-1629. doi:10.2147/idr.s249628

Korsmeyer, R. W., Gurny, R., Doelker, E., Buri, P., and Peppas, N. A. (1983). Mechanisms of Solute Release from Porous Hydrophilic Polymers. Int. J. pharm. 15 (1), 25-35. doi:10.1016/0378-5173(83)90064-9

Kumar, G. P., and Rajeshwarrao, P. (2011). Nonionic Surfactant Vesicular Systems for Effective Drug Delivery-An Overview. Acta pharm. sin. B 1 (4), 208-219. doi:10.1016/j.apsb.2011.09.002

Ladavière, C., and Gref, R. (2015). Toward an Optimized Treatment of Intracellular Bacterial Infections: Input of Nanoparticulate Drug Delivery Systems. Nanomedicine 10 (19), 3033-3055. doi:10.2217/nnm.15.128

Lajevardi, A., Hossaini Sadr, M., Tavakkoli Yaraki, M., Badiei, A., and Armaghan, M. (2018). A pH-Responsive and Magnetic Fe3O4@silica@MIL-100(Fe)/ $\beta$-CD Nanocomposite as a Drug Nanocarrier: Loading and Release Study of Cephalexin. New J. Chem. 42 (12), 9690-9701. doi:10.1039/c8nj01375f

Lawrence, M., Chauhan, S., Lawrence, S., and Barlow, D. (1996). The Formation, Characterization and Stability of Non-ionic Surfactant Vesicles. STP pharma Sci. 6 (1), 49-60.

Liang, X., Mao, G., and Ng, K. Y. S. (2004). Mechanical Properties and Stability Measurement of Cholesterol-Containing Liposome on Mica by Atomic Force Microscopy. J. Colloid Interf. Sci. 278 (1), 53-62. doi:10.1016/j.jcis.2004.05.042

Macià, M. D., Del Pozo, J. L., Díez-Aguilar, M., and Guinea, J. (2018). Microbiological Diagnosis of Biofilm-Related Infections. Enfermedades 
Infecciosas y Microbiologia Clinica (English ed.) 36 (6), 375-381. doi:10.1016/ j.eimce.2017.04.015

Manosroi, A., and Bauer, K. (1989). The Entrapment of a Human InsulinDEAE Dextran Complex in Different Compound Liposomes. Drug Dev. Ind. Pharm. 15 (14-16), 2531-2546. doi:10.3109/03639048909052545

Marianecci, C., Paolino, D., Celia, C., Fresta, M., Carafa, M., and Alhaique, F. (2010). Non-ionic Surfactant Vesicles in Pulmonary Glucocorticoid Delivery: Characterization and Interaction with Human Lung Fibroblasts. J. Controlled Release 147 (1), 127-135. doi:10.1016/j.jconrel.2010.06.022

Mirzaie, A., Peirovi, N., Akbarzadeh, I., Moghtaderi, M., Heidari, F., Yeganeh, F. E., et al. (2020). Preparation and Optimization of Ciprofloxacin Encapsulated Niosomes: A New Approach for Enhanced Antibacterial Activity, Biofilm Inhibition and Reduced Antibiotic Resistance in Ciprofloxacin-Resistant Methicillin-Resistance Staphylococcus aureus. Bioorg. Chem. 103, 104231. doi:10.1016/j.bioorg.2020.104231

Moammeri, A., Jamshidifar, E., Abbaspour, K., Sadeghi, S., Lamakani, L., and Akbarzadeh, I. J. B. C. (2021). Synergistic Effect of Curcumin-Cu and Curcumin-Ag Nanoparticle Loaded Niosome: Enhanced Antibacterial and Antibiofilm Activities. Bioorg. Chem. 115, 105116. doi:10.1016/j.bioorg.2021.105116

Moghaddam, F. D., Akbarzadeh, I., Marzbankia, E., Farid, M., Reihani, A. H., Javidfar, M., et al. (2021). Delivery of Melittin-Loaded Niosomes for Breast Cancer Treatment: an In Vitro and In Vivo Evaluation of Anti-cancer Effect. Cancer Nanotechnol. 12(1), 1-35. doi:10.1186/s12645-021-00085-9

Moghassemi, S., Parnian, E., Hakamivala, A., Darzianiazizi, M., Vardanjani, M. M., Kashanian, S., et al. (2015). Uptake and Transport of Insulin across Intestinal Membrane Model Using Trimethyl Chitosan Coated Insulin Niosomes. Mater. Sci. Eng. C 46, 333-340. doi:10.1016/j.msec.2014.10.070

Moghtaderi, M., Mirzaie, A., Zabet, N., Moammeri, A., Mansoori-Kermani, A., Akbarzadeh, I., et al. (2021). Enhanced Antibacterial Activity of Echinacea Angustifolia Extract against Multidrug-Resistant Klebsiella pneumoniae through Niosome Encapsulation. Nanomaterials 11 (6), 1573. doi:10.3390/nano11061573

Mugabe, C., Azghani, A. O., and Omri, A. (2005). Liposome-mediated Gentamicin Delivery: Development and Activity against Resistant Strains of Pseudomonas aeruginosa Isolated from Cystic Fibrosis Patients. J. Antimicrob. Chemother. 55 (2), 269-271. doi:10.1093/jac/dkh518

Paolino, D., Cosco, D., Muzzalupo, R., Trapasso, E., Picci, N., and Fresta, M. (2008). Innovative Bola-Surfactant Niosomes as Topical Delivery Systems of 5fluorouracil for the Treatment of Skin Cancer. Int. J. Pharm. 353 (1-2), 233-242. doi:10.1016/j.jipharm.2007.11.037

Pardakhty, A., Moazeni, E., Varshosaz, J., Hajhashemi, V., and Rouholamini Najafabadi, A. (2011). Pharmacokinetic Study of Niosome-Loaded Insulin in Diabetic Rats. Daru 19 (6), 404-411.

Raza, M., Kanwal, Z., Rauf, A., Sabri, A., Riaz, S., and Naseem, S. (2016). Size- and Shape-dependent Antibacterial Studies of Silver Nanoparticles Synthesized by Wet Chemical Routes. Nanomaterials 6 (4), 74. doi:10.3390/nano6040074

Reta, A., Bitew Kifilie, A., and Mengist, A. (2019). Bacterial Infections and Their Antibiotic Resistance Pattern in Ethiopia: A Systematic Review. Adv. Prev. Med. 2019, 4380309. doi:10.1155/2019/4380309

Ruckmani, K., and Sankar, V. (2010). Formulation and Optimization of Zidovudine Niosomes. Aaps Pharmscitech 11 (3), 1119-1127. doi:10.1208/ s12249-010-9480-2

Ruckmani, K., Jayakar, B., and Ghosal, S. K. (2000). Nonionic Surfactant Vesicles (Niosomes) of Cytarabine Hydrochloride for Effective Treatment of Leukemias: Encapsulation, Storage, and In Vitro Release. Drug Dev. Ind. Pharm. 26 (2), 217-222. doi: $10.1081 /$ ddc- 100100348

Sadeghi, S., Bakhshandeh, H., Ahangari Cohan, R., Peirovi, A., Ehsani, P., and Norouzian, D. (2019). Synergistic Anti-staphylococcal Activity of Niosomal Recombinant Lysostaphin-LL-37. Ijn 14, 9777-9792. doi:10.2147/ijn.s230269

Sadeghi, S., Ehsani, P., Cohan, R. A., Sardari, S., Akbarzadeh, I., Bakhshandeh, H., et al. (2020). Design and Physicochemical Characterization of Lysozyme Loaded Niosomal Formulations as a New Controlled Delivery System. Pharm. Chem. J. 53 (10), 921-930. doi:10.1007/s11094-020-02100-6

Samiei, M., Farjami, A., Dizaj, S. M., and Lotfipour, F. (2016). Nanoparticles for Antimicrobial Purposes in Endodontics: A Systematic Review of In Vitro Studies. Mater. Sci. Eng. C 58, 1269-1278. doi:10.1016/j.msec.2015.08.070
Shabani, A., Atyabi, F., Khoshayand, M. R., Mahbod, R., Cohan, R. A., Akbarzadeh, I., et al. (2020). Design of experiment, Preparation, and In Vitro Biological Assessment of Human Amniotic Membrane Extract Loaded Nanoparticles. Cpb 21 (3), 256-267. doi:10.2174/1389201020666191019122130

Shad, P. M., Karizi, S. Z., Javan, R. S., Mirzaie, A., Noorbazargan, H., Akbarzadeh, I., et al. (2020). Folate Conjugated Hyaluronic Acid Coated Alginate Nanogels Encapsulated Oxaliplatin Enhance Antitumor and Apoptosis Efficacy on Colorectal Cancer Cells (HT29 Cell Line). Toxicol. Vitro 65, 104756. doi:10.1016/j.tiv.2019.104756

Shaker, D. S., Shaker, M. A., and Hanafy, M. S. (2015). Cellular Uptake, Cytotoxicity and In-Vivo Evaluation of Tamoxifen Citrate Loaded Niosomes. Int. J. Pharm. 493 (1-2), 285-294. doi:10.1016/j.jipharm.2015.07.041

Sharma, D., Cukras, A. R., Rogers, E. J., Southworth, D. R., and Green, R. (2007). Mutational Analysis of S12 Protein and Implications for the Accuracy of Decoding by the Ribosome. J. Mol. Biol. 374 (4), 1065-1076. doi:10.1016/j.jmb.2007.10.003

Shirzad, M., Jamehbozorgi, S., Akbarzadeh, I., Aghabozorg, H. R., Amini, A., and technologies, d. d. (2019). The Role of Polyethylene Glycol Size in Chemical Spectra, Cytotoxicity, and Release of PEGylated Nanoliposomal Cisplatin. ASSAY Drug Dev. Tech. 17 (5), 231-239. doi:10.1089/adt.2019.923

Shrestha, A., and Kishen, A. (2016). Antibacterial Nanoparticles in Endodontics: a Review. J. Endod. 42 (10), 1417-1426. doi:10.1016/j.joen.2016.05.021

Tarekegn, A., Joseph, N. M., Palani, S., Zacharia, A., and Ayenew, Z. (2010). Niosomes in Targeted Drug Delivery: Some Recent Advances. IJPSR 1 (9), 1-8.

Taymouri, S., and Varshosaz, J. (2016). Effect of Different Types of Surfactants on the Physical Properties and Stability of Carvedilol Nano-Niosomes. Adv. Biomed. Res. 5, 48. doi:10.4103/2277-9175.178781

Vikas, K., Arvind, S., Ashish, S., Gourav, J., and Vipasha, D. (2011). Recent Advances in NDDS (Novel Drug Delivery System) for Delivery of Antihypertensive Drugs. Int. J. Drug Dev. Res. 3 (1), 252-259.

Waddad, A. Y., Abbad, S., Yu, F., Munyendo, W. L. L., Wang, J., Lv, H., et al. (2013). Formulation, Characterization and Pharmacokinetics of Morin Hydrate Niosomes Prepared from Various Non-ionic Surfactants. Int. J. pharm. 456 (2), 446-458. doi:10.1016/j.ijpharm.2013.08.040

de Lima Procópio, R. E., da Silva, I. R., Martins, M. K., de Azevedo, J. L., and de Araújo, J. M. (2012). Antibiotics Produced by Streptomyces. Braz. J. Infect. Dis. 16 (5), 466-471. doi:10.1016/j.bjid.2012.08.014

Zhang, L., Gu, F., Chan, J., Wang, A., Langer, R., and Farokhzad, O. (2008). Nanoparticles in Medicine: Therapeutic Applications and Developments. Clin. Pharmacol. Ther. 83 (5), 761-769. doi:10.1038/sj.clpt.6100400

Zhu, M., Burman, W. J., Jaresko, G. S., Berning, S. E., Jelliffe, R. W., and Peloquin, C. A. (2001). Population Pharmacokinetics of Intravenous and Intramuscular Streptomycin in Patients with Tuberculosis. Pharmacotherapy 21 (9), 1037-1045. doi:10.1592/phco.21.13.1037.34625

Zille, A., Fernandes, M. M., Francesko, A., Tzanov, T., Fernandes, M., Oliveira, F. R., et al. (2015). Size and Aging Effects on Antimicrobial Efficiency of Silver Nanoparticles Coated on Polyamide Fabrics Activated by Atmospheric DBD Plasma. ACS Appl. Mater. Inter. 7 (25), 13731-13744. doi:10.1021/acsami.5b04340

Conflict of Interest: The authors declare that the research was conducted in the absence of any commercial or financial relationships that could be construed as a potential conflict of interest.

Publisher's Note: All claims expressed in this article are solely those of the authors and do not necessarily represent those of their affiliated organizations, or those of the publisher, the editors, and the reviewers. Any product that may be evaluated in this article, or claim that may be made by its manufacturer, is not guaranteed or endorsed by the publisher.

Copyright (c) 2021 Mansouri, Khayam, Jamshidifar, Pourseif, Kianian, Mirzaie, Akbarzadeh and Ren. This is an open-access article distributed under the terms of the Creative Commons Attribution License (CC BY). The use, distribution or reproduction in other forums is permitted, provided the original author(s) and the copyright owner(s) are credited and that the original publication in this journal is cited, in accordance with accepted academic practice. No use, distribution or reproduction is permitted which does not comply with these terms. 